\title{
Modification of polymers for cardiovascular applications-some routes to bioactive hydrophilic polymers
}

\author{
C H BAMFORD, I P MIDDLETON, K G AL-LAMEE, J PAPROTNY \\ and Y SATAKE \\ Institute of Medical and Dental Bioengineering, Duncan Building, University of Liverpool, \\ P O Box 147, Liverpool L69 3BX, England
}

\begin{abstract}
This paper is concerned with the activation of platelets by polymers, a keyprocess in the behaviour of prosthetic devices in contact with blood.

Platelets are activated by contact with many different types of polymer surfaces, which must therefore be regarded as thrombogenic. Two procedures for reducing thrombogenicity are discussed: (i) the chemical attachment of inhibitors of platelet aggregation and (ii) gross modification of the nature of the surface, e.g. by making it more hydrophilic. For purposes of (i) the potent prostaglandin analogue $\mathrm{BW} 245 \mathrm{C}$ has been used, while for (ii) grafting of poly(ethylene glycol) (PEG) has been explored. Both methods give greatly reduced platelet adhesion in in vitro tests.

The second part of the paper deals with the properties of adducts of inhibitors of platelet aggregation (BW 245C, dipyridamole) with water-soluble macromolecules [poly(N-viny] pyrrolidone), PEG, dextran]. Adducts have been synthesized with terminal and side-chain coupling. On adduction the two inhibitors mentioned show opposite types of behaviour: the molar activity of BW $245 \mathrm{C}$ is dramatically reduced, but that of dipyridamole is significantly increased. Remarkable synergistic effects have been recorded for BW $245 \mathrm{C}$ adducts. These observations are interpreted in terms of differences in stereochemistry in the drug-receptor interactions.

Appropriate chemical techniques for coupling are outlined, attention being drawn to the special uses of haloalkyl- and haloacyl-isocyanates and 2-isocyanatoethyl methacrylate as reagents.
\end{abstract}

Keywords. Platelet adhesion and aggregation; prostaglandin analogue; BW 245C; dipyridamole; poly(ether urethane urea); polystyrene; poly(N-vinyl pyrrolidone); poly (ethylene glycol); dextran; non-thrombogenic surface.

\section{Introduction}

In the cardiovascular system nature has achieved a self-regulating mechanism of great beauty and complexity. Damage to a blood-vessel which causes the constituent collagen to be exposed brings about rapid activation of blood platelets in the neighbourhood, with initiation of chemical changes directed towards sealing off the injured area, which becomes the site of platelet adhesion and aggregation. Thromboxane (1) is liberated in the activated platelets and in turn stimulates processes which include activation of further platelets and calcium release from the store in the platelet granules. The ensuing clumping together of platelets to form aggregates is the first stage in the formation of a stable blood clot in the region of the injury.

The action of thromboxane is opposed by that of a prostaglandin-prostacyclin $\left(\mathrm{PGI}_{2}\right)(2)$-synthesized within the vessel walls; this stimulates the enzyme adenylate cyclase in the platelets to form cyclic adenosine monophosphate (c-AMP) which blocks the action of thromboxane and so powerfully inhibits platelet adhesion and 
1

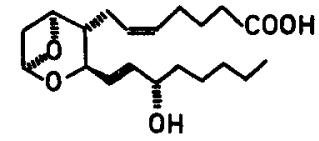

Thromboxane $\mathrm{A}_{2}$

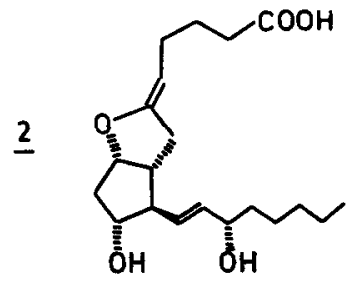

Prostacyclin $\left(\mathrm{PGI}_{2}\right)$

aggregation. Under normal conditions very small quantities of prostaglandin are secreted by the vessel walls and may play a vital role in maintaining stable equilibrium between the walls and the blood. Prostacyclin has a short half-life (of the order of minutes) in the circulation, but the very low concentrations existing in the blood discourage significant and progressive aggregation of platelets such as might follow damage to these cells by turbulence in the blood-stream, for example.*

Although collagen is the most potent activator of platelets, other substances including many polymers can also behave in this fashion and so initiate thrombusformation. While there is at present no accepted order of thrombogenicity-indeed it has proved difficult to quantify this property in any general way-it is unfortunately the case that many common polymers (e.g. polystyrene) must be qualitatively classed as thrombogenic.

Replacement of a natural vessel by a prosthesis constructed of polymer, must therefore at best (given a relatively non-thrombogenic material) reduce the local state of vessel wall/blood equilibrium from stable or metastable to unstable, since one component in the equilibrium is almost eliminated. Additionally, the anastomoses are potentially sites of thrombus-formation and occlusion since collagen is likely to be exposed there.

In time, if no catastrophic platelet aggregation occurs, new endothelial cells may grow across the prosthesis from the ends, or natural growth may occur through the pores of the prosthesis if they are sufficiently large (Clowes et al 1986), and prostacyclin secretion again becomes possible. When this situation is reached occlusion is evidently less probable. Occlusion appears to occur much more easily in tubes of narrow bore-less than about $4 \mathrm{~mm}$ in diameter. It is commonly thought that blood turbulence increases the tendency towards thrombus-formation and this is likely to be particularly important in narrow crimped tubes.

Non-thrombogenicity, although obviously of great importance, is not the only property which a polymer must possess if it is to be used successfully in an arterial prosthesis. The elastic properties would be expected to be important, and intuitively a match with the corresponding properties of the natural vessel seems desirable. Lyman et al (1978) have remarked "We believed that the mismatch in compliance between the polymer and the vessel was causing sufficient trauma to the natural vessel to disrupt the endothelium and thus initiate thrombosis".

A third consideration, also of major importance, is the attack on the polymer by local tissues with which it is in contact, leading to either chemical (e.g. hydrolytic)

\footnotetext{
* Recent measurements have suggested that circulating levels may be too low to allow prostacyclin to perform this regulatory function in the blood serum (Chesterman et al 1986).
} 
or enzymic degradation. Obviously such degradation, if significant, would weaken the prosthesis and could result in its mechanical failure.

Poly(ether urethane)s and poly(ether urethane urea)s have been much favoured in experimental work on small-bore arterial prostheses by virtue of their good mechanical properties (including high tensile strength and high ultimate elongation at break) and relatively good biocompatibility. However, although their performance as a whole is probably better than that of most other polymers, they are not free from defects of the types discussed above. Indeed, it is arguable that no "simple" synthetic polymer is likely to be found in the near future with the ideal spectrum of properties. The remainder of this paper is concerned with the improvement of polymer haemocompatibility by chemical modification.

\section{Anti-platelet aggregation agents}

Following the model of the natural vessels, we may expect incorporation in the walls of the prosthesis of an inhibitor of platelet aggregation, especially a prostaglandin or analogue, to improve the haemocompatibility by tending to restore the equilibrium state referred to earlier. "Incorporation" may range from simple mixing to chemical binding, either by labile or stable links.

We have used the potent prostaglandin analogue BW $245 \mathrm{C}$ (3) kindly donated to us by Dr Norman Whittaker of the Wellcome Research Laboratories. The most active diastereomer of this compound, which has approximately one-quarter of the inhibitory activity of prostacyclin, is far more potent than other prostaglandins which inhibit aggregation (Brockwell et al 1981; Whittle et al 1983). We have shown that a catheter coated with polystyrene in which (3) had been dispersed when placed in the vein of a dog accumulated no thrombus in the course of four hours, while a similar catheter, coated with polystyrene without (3) became covered in thrombus. In principle, therefore, this procedure of mimicking $\mathrm{PGI}_{2}$ secretion appears to work. Before it could be applied therapeutically much investigation would be required to establish that the rate of diffusion of the anti-platelet agent is commensurate with the length of time during which the prosthesis must be protected against platelet adhesion. It is becoming evident that occlusion is most likely to occur early, so that this technique, which would probably be effective for a few days, may be useful. The general effect of introducing the anti-platelet agent into the circulation would obviously need careful exploration.

BW 245 C

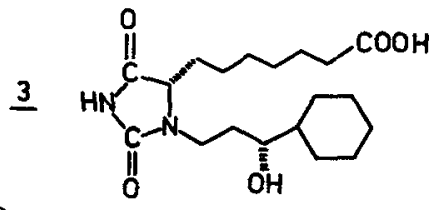

There have been numerous reports of experiments intended to attach anti-platelet agents to polymers by chemical bonds, notably one by Ebert et al (1982), on the binding of prostacyclin to polystyrene. In most cases, chemical procedures have contained some ambiguity so that the results have not been decisive. We have endeavoured to use well-defined chemical routes in synthesizing adducts of 
BW 245C and polymers. Our work on coupling of this drug to the poly(ether urethane urea) Biomer has already been reported (Bamford and Middleton 1983; Bamford et al 1985) and will be outlined here only briefly. We decided to use freeradical reactions for the coupling of polymerizable derivatives of $\mathrm{BW} 245 \mathrm{C}$. For this purpose Biomer is functionalised by converting some urethane groups to N-chloroor N-bromo-derivatives; on reaction (photochemical or thermal) with a transition metal complex — NCOO_ radicals are generated (Bamford 1974) and are sufficiently active to initiate polymerization of vinyl monomers (scheme 1). This procedure is applicable to a wide range of poly(ether urethane)s and is convenient for synthesizing graft copolymers (Bamford and Middleton 1983). Appropriate functionalization of BW $245 \mathrm{C}$ to convert it into a vinyl monomer may be effected through the carboxyl group; the hydroxyl at $15 \mathrm{C}$ must be left unchanged if activity is to be preserved, and substitution in the $\mathrm{NH}<$ leads to great loss of activity (Bamford et al 1985). The methacrylate hydroxy-esters shown in (4) were used to esterify the drug. Polymerization of the resulting 245C-monomers yields polymers in which (3) is attached in side chains of lengths determined by $n$ and $m$ and with hydrophilic or hydrophobic properties, respectively. Clearly these $245 \mathrm{C}$-monomers may be copolymerized with common vinyl monomers and their attachment to poly(ether-urethane)s either as homo- or co-polymers, through the reactions summarised in scheme 1 , needs no further comment.

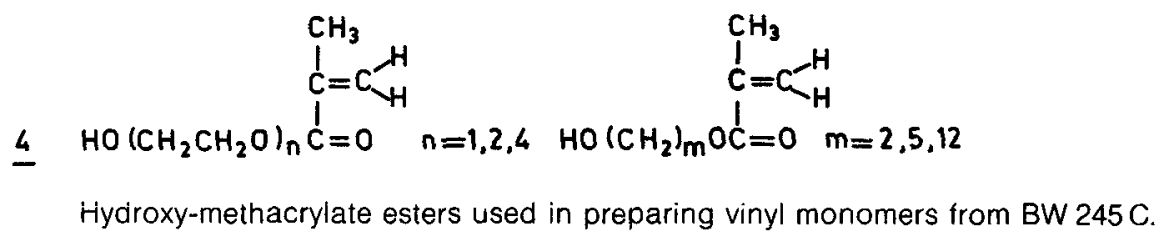

Biomer treated in this way carrying grafts of a copolymer of $\mathrm{N}$-vinyl pyrrolidone and 245C-monomer [derived from (4), $n=1$ ] (BW 245C content $2.68 \% \mathrm{w} / \mathrm{w}$ of whole) was cast into films from dimethylacetamide and examined for platelet adhesion (Bamford et al, in preparation). Figure 1 indicates that platelet adhesion to this film was very much less than that with a film of pure Biomer. Similar findings are shown in figure 2 for a copolymer of styrene and the $245 \mathrm{C}$-monomer containing $1.85 \% \mathrm{w} / \mathrm{w}$ of BW $245 \mathrm{C}(0.4 \%$ molar) (5). At the end of these experiments the plasma had no anti-aggregatory action, indicating that the drug had not been detached from the polymer in significant quantity. J C Barbenel (private communication) employed a

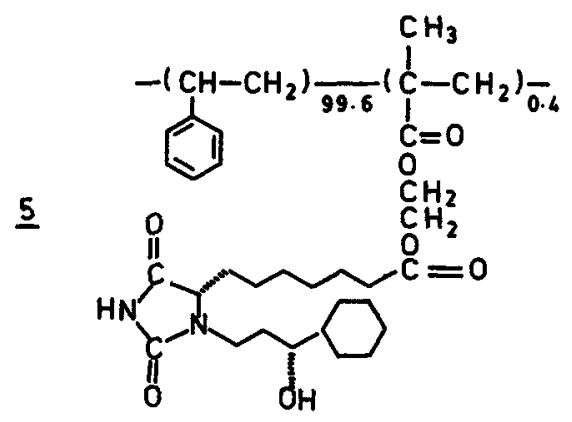

BW 245 C Polystrene adduct. 


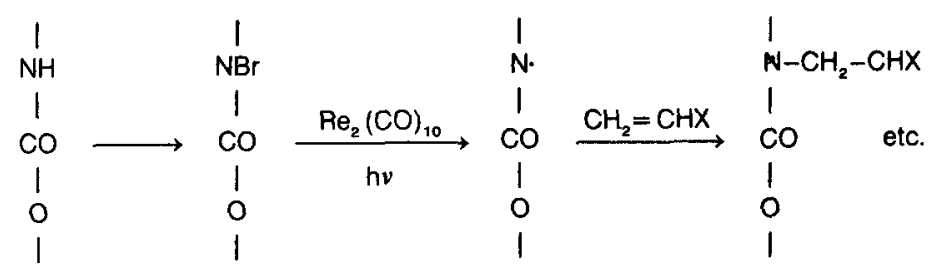

Scheme 1. Synthesis of graft copolymers of polyurethanes by free-radical reactions.
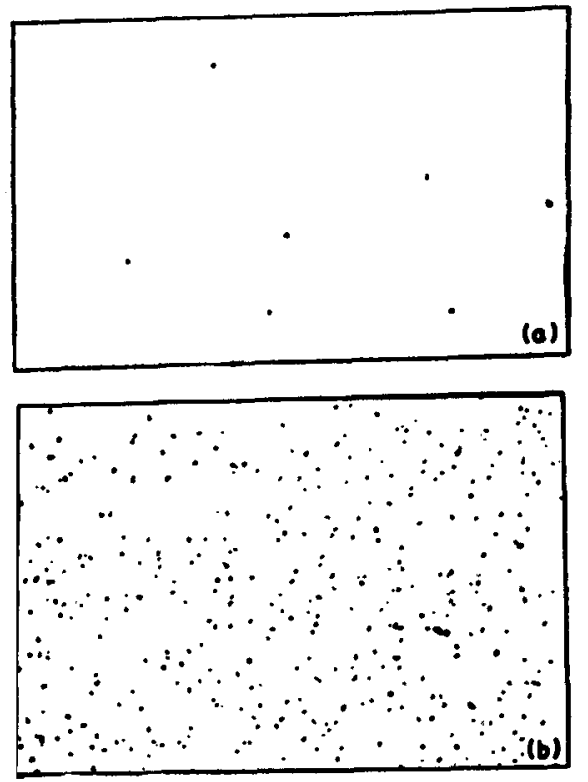

Figure 1. Blood-platelet adhesion to polymer films. (a) Biomer with grafted copolymer of $\mathrm{N}$-vinyl pyrrolidone and 245C-monomer (derived from $\underline{4}, n=1$ ). BW $245 \mathrm{C}$ content $2.68 \% \mathrm{w} / \mathrm{w}$ of total. (b) Pure Biomer. The films cast from dimethylacetamide were immersed in sheep platelet-rich-plasma for $4 \mathrm{~h}$ at ambient temperatures, washed and stained.

modification of the $\mathrm{Wu}$ and Hoak (1974) technique to compare the interactions of human blood platelets with a copolymer of methyl acrylate and styrene and a similar copolymer containing in addition BW 245C monomer units (BW 245C content $1.5 \% \mathrm{w} / \mathrm{w})$. Platelet counts made in whole blood exposed to each of the test surfaces for 3,6 or 9 min showed that platelet adhesion and aggregation were significantly reduced in blood exposed to surfaces containing BW 245C.

These experiments indicate that chemical attachment of BW $245 \mathrm{C}$ to polymers substantially reduces platelet adhesion; however, such encouraging results have not yet been obtained in vivo. A catheter covered with the Biomer film to be tested was passed upwards through a femoral vein into the external iliac vein of a dog, while a similar catheter coated with the same polymer without $245 \mathrm{C}$ was inserted into the second external iliac vein to act as blank. The development of thrombus, assessed by use of In radio-labelled platelets, was similar on the two catheters.

There are important differences between the in vitro and in vivo experiments. (i) Platelet-rich-plasma for the former is normally isolated with the aid of citrate buffer, which inhibits blood clotting involving fibrin, a process which may well play a major role in the formation of thrombus in vivo. (ii) There is a possibility in vivo of platelet damage arising from turbulence or chance contact with collagen 

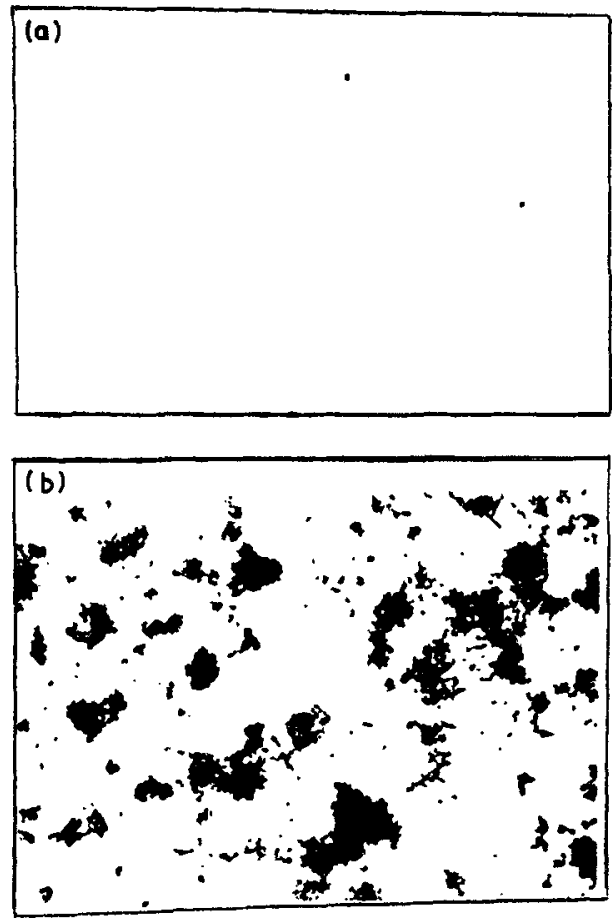

Figure 2. Blood-platelet adhesion to polymer films. (a) Copolymer of styrene and 245C-monomer shown in 5 . (b) Pure polystyrene. Technique as described for figure 1.

contaminating the catheter etc. Thus, it may be necessary for the anti-platelet agent to diffuse some short distance from the vessel walls and set up a protective layer, as in the natural system. In this event, slow diffusion of the anti-platelet agent out of the polymer rather than permanent attachment would be required. We have found that heparinization will prevent accumulation of thrombus on catheters in the type of experiment described above. Uziel et al (1986) reported that minimal doses of heparin with prostacyclin infusion greatly reduce platelet aggregation and maintain high platelet counts during extracorporeal circulation in sheep; however $\mathbf{P G I}_{2}$ in the absence of heparin was found to be ineffective. These results suggest that a study of the interaction of blood with polymer surfaces carrying both an antiplatelet agent and heparin would be rewarding.

There is evidently a need for much further investigation of these and related matters.

\section{Hydrophilic polymers}

There has been some debate in the literature about possible merits of introducing hydrophilic grafts for increasing the haemocompatibility of polymers (Ratner et al 1979). Sung and $\mathrm{Hu}$ (1979) studied by ESCA Biomer films cast in air and showed that the air-facing surface contained a higher proportion of soft segment. Sa da Costa et al (1981) examined several segmented poly(ether-urethane-urea)s with different hard and soft segments, employing glass beads coated with polymer and measuring the mean platelet retention index $\rho$ for human blood from six different 
donors. The order of increasing $\rho$ for the polyether component in the soft segments was found to be:

$$
\text { poly (ethylene oxide) < poly (propylene oxide) < poly (tetramethylene oxide). }
$$

Sa da Costa et al (1981) concluded that "amorphous cross-linked poly(ethylene oxide) might be a remarkably bland material of interest for prolonged blood contact."

Bantjes and his colleagues (Bots et al 1986) have followed up this finding by constructing narrow-bore vascular grafts of cross-linked copolymers of poly(ethylene oxide) and poly(propylene oxide) and have reported promising results in experiments on rats. We have grafted poly(ethylene glycol) (PEG) chains to Biomer in an attempt to make a polymer in which the good mechanical properties of Biomer and the low platelet retention index of poly(ethylene oxide) are retained.

At this point we digress to discuss synthetic routes, which so far as we are aware have not been used previously by others, but which promise to be valuable in the synthesis of complex polymers. For the synthesis of Biomer-PEG grafts set out in scheme 2 we converted Biomer into a macroinitiator using 2-chloroethyl isocyanate (6) and synthesized a macromer from PEG by reaction with 2-isocyantoethyl methacrylate (7). (Both isocyanates are commercial products; haloalkyl and haloacyl isocyanates have been used as derivatising agents for over two decades.) The haloisocyanate reacts readily with polyurethanes in dimethylacetamide solution at ambient temperatures, forming allophanate derivatives ( $\underline{8}$ ), the 2-chloroethyl

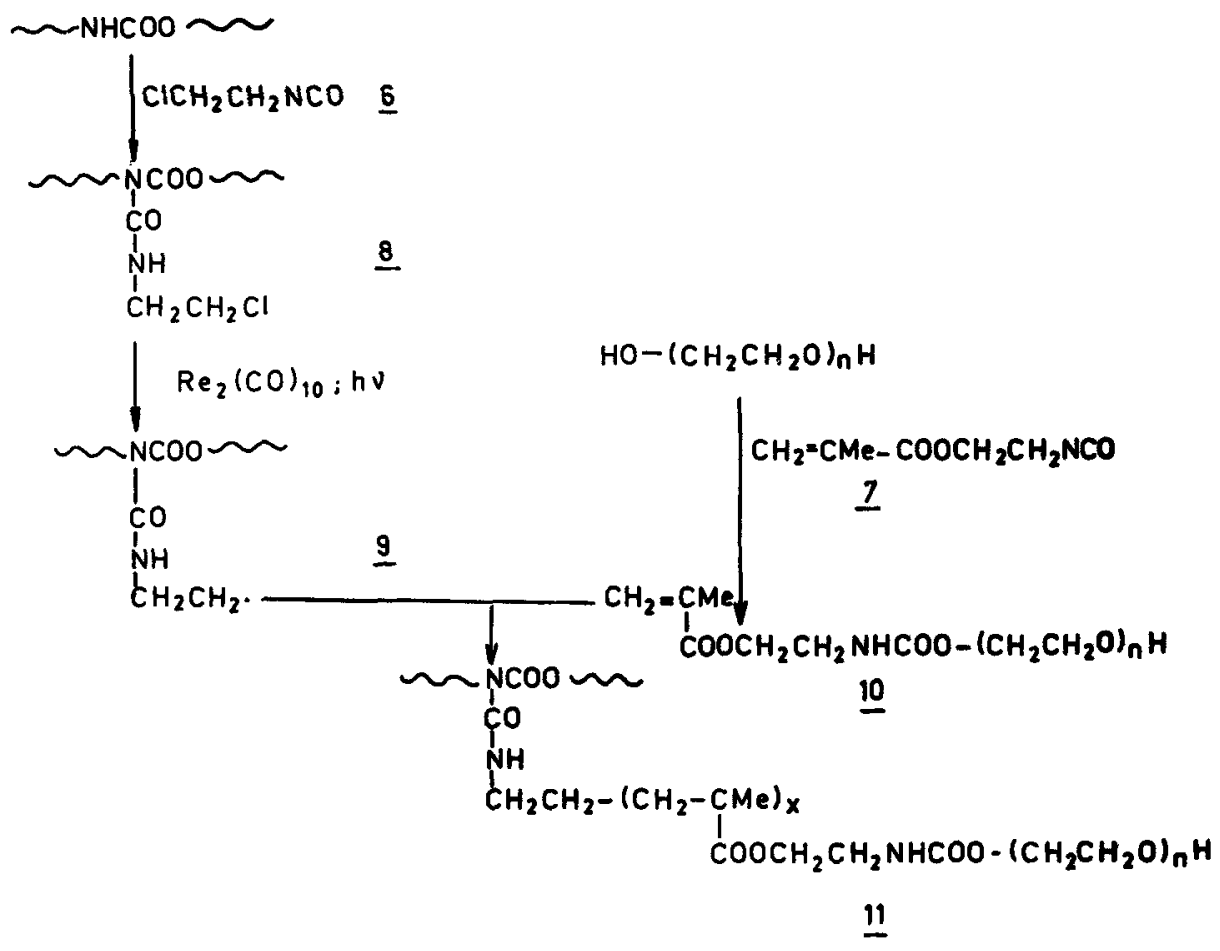

Scheme 2. Synthesis of graft copolymers of polyurethanes and poly(ethylene glycol). 
moiety of which initiates radicals $(\underline{9})$ on reaction with a suitable transition metal complex [e.g. $\operatorname{Re}_{2}(\mathrm{CO})_{10}$ ]. This method of functionalization of Biomer is an alternative to that outlined in scheme 1. Poly (ethylene glycol) after conversion to a polymerizable vinyl macromer (10) ultimately yields a Biomer-PEG graft of structure (11).

Films of these grafts containing $8 \%$ and $33 \%$ PEG-4000 cast from dimethylacetamide were tested for platelet adhesion as described for BiomerBW 245C grafts (see figure 1). Photographs of representative areas of the films showing the adhered platelets (figure 3) clearly indicate that grafting of PEG greatly reduces platelet adhesion to Biomer.

We intend to extend this type of study to grafts of other water-soluble polymers and to in vivo experiments.

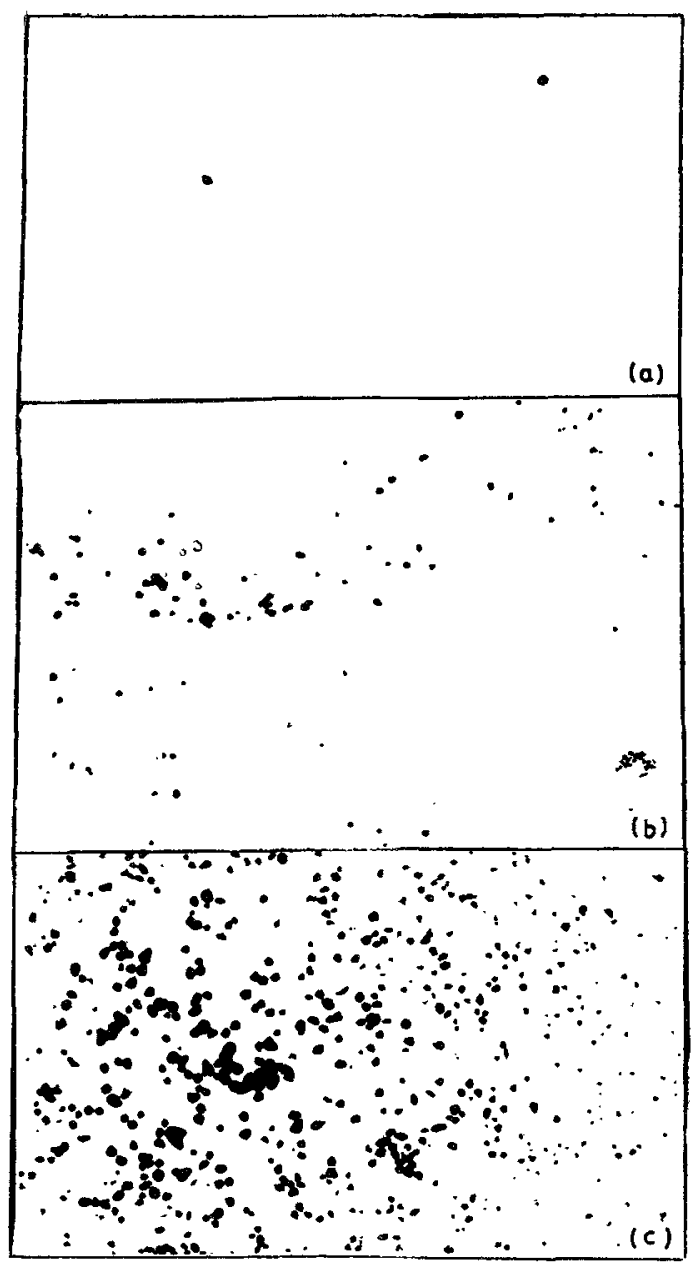

Figure 3. Blood-platelet adhesion to polymer films. (a) Biomer-PEG-4000 graft copolymer 11. PEG content $33 \% \mathrm{w} / \mathrm{w}$. (b) Biomer-PEG-4000 graft copolymer 11 . PEG content $8 \% \mathrm{w} / \mathrm{w}$. (c) For pure Biomer see figure $1 \mathrm{~b}$. Technique as described for figure 1 . 


\section{Water-soluble polymers}

Investigations of the types hitherto considered are valuable in so far as they illustrate practical methods by which the haemocompatibility of polymers may be improved, but they cast relatively little light on the fundamentals of polymer-platelet interaction. It seemed to us that systematic investigations of platelet aggregation employing water-soluble polymers carrying bound drugs would produce results capable of more detailed interpretation. We now describe relevant work in which aggregation of sheep platelet-rich plasma [generally induced by addition of adenosine diphosphate (ADP)] was followed by conventional Born aggregatory. We believe that such observations could contribute fundamental data required for the design of non-thrombogenic polymers.

\subsection{Adducts of $B W 245 C$ with polymers}

BW 245C was coupled to water-soluble polymers in three ways (Bamford et al 1986a, c). (a) The BW 245C-monomers based on the methacrylate hydroxy esters in (4) were copolymerized with $\mathrm{N}$-vinyl pyrrolidone (NVP) to yield drug moieties coupled in side-chains; (b) An ester of (3) with a halo-alcohol such as $\mathrm{HOCH}_{2} \mathrm{CCl}_{3}$ or $\mathrm{HOCH}_{2} \mathrm{CH}_{2} \mathrm{Br}$ in association with a metal carbonyl is a free-radical initiator which was used to attach terminal groups of (3) to a vinyl polymer chain; (c) Both side-chain and terminal incorporation of (3) may also be achieved by direct esterification with the hydroxyl groups of a water-soluble polymer such as PEG or dextran (Bamford et al 1986a) (see $\underline{1} \underline{2}$ and 13 ).

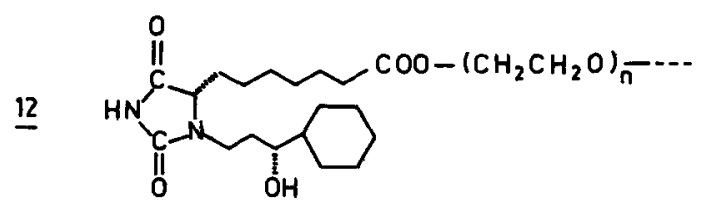

BW 245 C-poly (ethylene glycol) adduct.

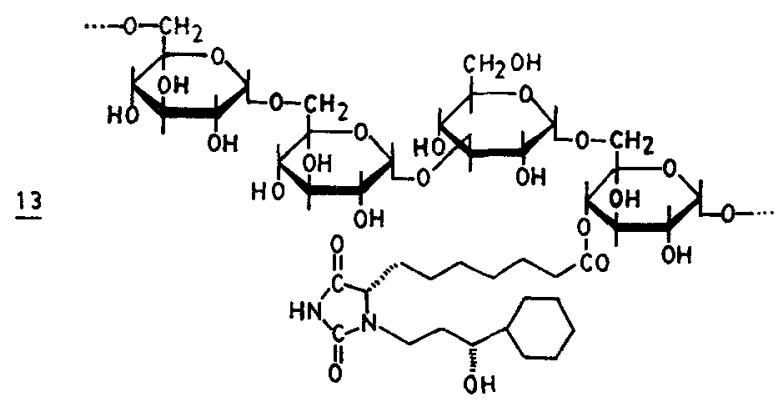

Portion of dextran chain with attached BW 245 C.

Esterification of the carboxyl group in BW $245 \mathrm{C}$ by a low-molecular-weight alcohol produces a 10-20 fold reduction in activity which is rather insensitive to the nature of the esterifying group in our experiments (figure 4). The activity of 


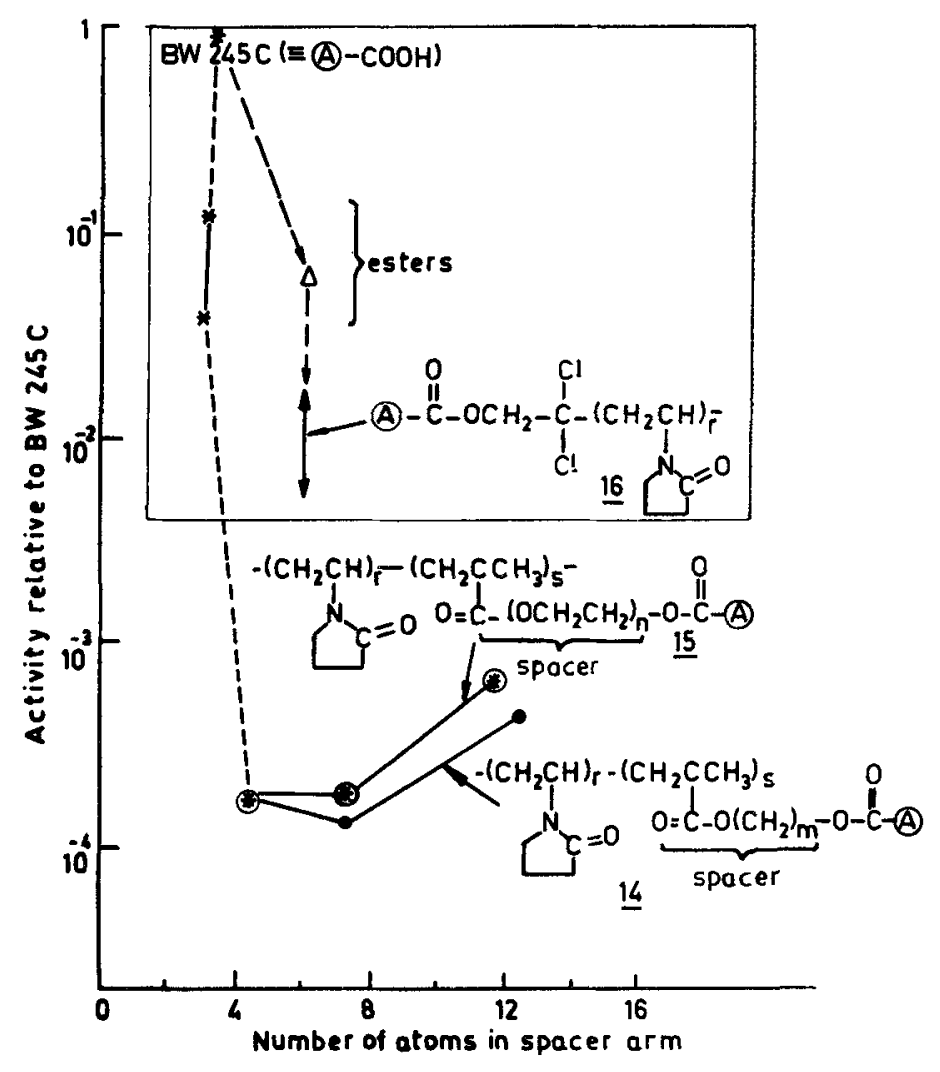

Figure 4. Molar activities of $\mathrm{BW} 245 \mathrm{C}$ as platelet aggregation inhibitor in water-soluble polymer adducts. *-* BW 245C monomers; $\triangle \mathrm{BW} 245 \mathrm{C}$ haloesters.

BW 245C falls greatly when coupled to poly NVP, but the decrease is determined by the position of the drug molecule in the polymer chain (Bamford et al 1986c). The results are summarised in figure 4 (Bamford et al 1987c). Side-chain coupling produces the greatest reduction, by approximately a factor of $10^{3}-10^{4}$ on a molar basis. The side-chain (spacer arm) length and hydrophilicity have minor influences on the activity [figure 4, polymers (14) and (1ㄷ)]. Coupling as a terminal group leads to smaller reductions, approximately 100 -fold [polymer (16)]. A remarkable synergistic effect was revealed initially (Bamford et al 1986b) by studies of adducts with PEG (12 2 , mol. wt. 10,000) and dextran (13, mol. wt. 71,200); the molar activity of the coupled drug was found to be greatly enhanced when the adduct was mixed with the uncoupled parent polymer. The latter alone has no significant effect in the concentrations used. Data are presented in table 1 which show that the enhancement can be very large - 100 -fold with the dextran adduct. Although the increase is less with PEG $(10,000)(10$-fold) the activity of BW $245 \mathrm{C}$ in the " $0.01 \%$ " adduct is remarkably high, nearly $50 \%$ of that of the free drug and in fact higher than that of any simple ester examined. Another feature illustrated in table 1 is the rather sharp molecular weight dependence, the enhancement observed with PEG (4000) being much smaller than that with PEG $(10,000)$. A third characteristic is the "cross- 
polymer" effect: the activity of BW $245 \mathrm{C}$ attached to one species of polymer chain is enhanced only to small extents by "dilution" with a different polymer.

We believe that all these phenomena can be interpreted in terms of steric interference in the drug-receptor interaction. The chemical changes in the BW $245 \mathrm{C}$ molecules produced by coupling to polymer chains in our experiments are the same as those arising from esterification with low-molecular-weight alcohols; further, we have already noted that the character of the alcohol has little effect on the activity of the ester (cf. figure 4). Thus it is reasonable to attribute the reductions in activity in figure 4 to steric, rather than chemical, influences, particularly to interferences between the chains and the lipid bilayers which impede access of the drug to its receptor. This is in keeping with the observation that the activity is greater for terminal than side-chain coupling (figure 4). The phenomena reported in table 1 can be interpreted in terms of adsorption of (inactive) polymer on to platelet membranes, which thereby became disorganized, particularly near the glyco-protein components on which receptors are located. The receptors thus become less subject to steric obstruction or "externalized". This type of mechanism, based on physical processes, is consistent with all the experimental data.

Very different results (Bamford et al 1987a) are obtained with the vasodilator dipyridamole (17), which is also an inhibitor of platelet aggregation. This drug is
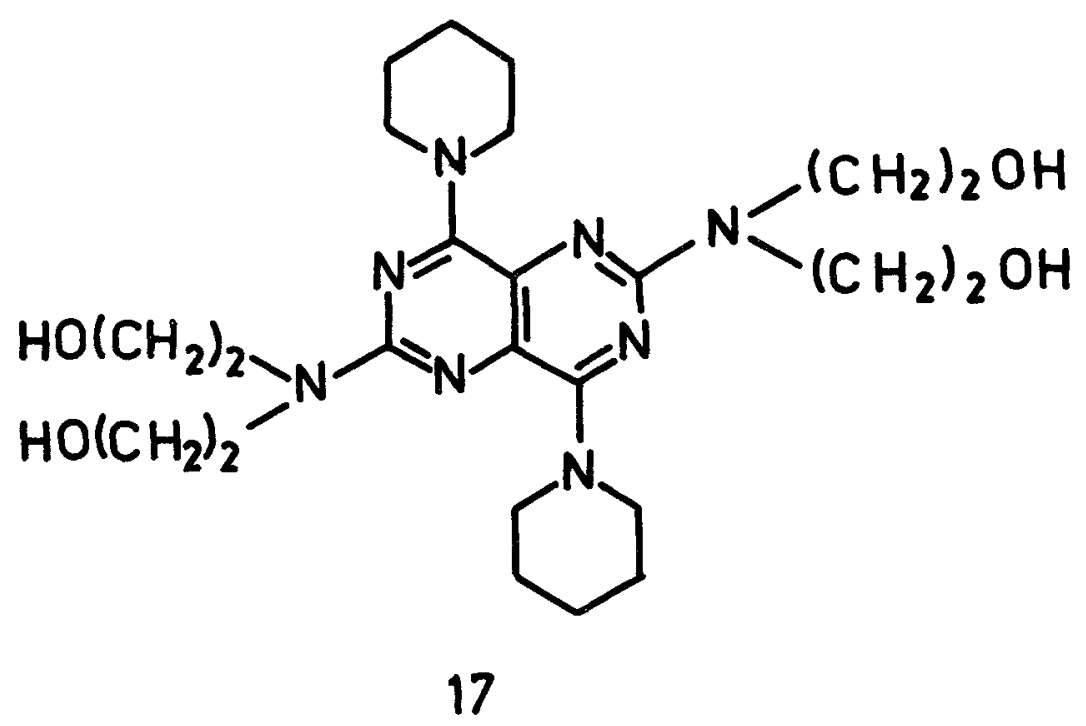

Dipyridamole

much less demanding in its steric requirements for interaction with its receptor, indeed one piperidine ring may be removed without major changes in activity. By preparing the monomethacrylate ester of (1ㄱ) we have converted the latter into a vinyl monomer (DIMA) which we have copolymerized with $\mathrm{N}$-vinylpyrrolidone. The inhibitory activities of the monomer and polymer species are presented in table 2.

These data contrast strikingly with those for BW 245C systems (table 1) since the dipyridamole/polymer adducts are more active than the free drug as aggregation 
Table 1. Activities of BW $245 \mathrm{C}$ in polymer-drug adducts.

\begin{tabular}{|c|c|c|c|}
\hline \multirow[b]{2}{*}{$\begin{array}{l}\text { Content of BW } 245 C \\
\text { in adduct }(\% \mathrm{w} / \mathrm{w})\end{array}$} & \multicolumn{2}{|c|}{$\begin{array}{l}\text { Weights for } 50 \% \\
\text { Inhibition }(\mu \mathrm{g} / \mathrm{ml})\end{array}$} & \multirow[b]{2}{*}{$\begin{array}{c}\text { Relative activity } \\
\text { of coupled BW 245C }\end{array}$} \\
\hline & $\begin{array}{c}\text { BW } 245 C \\
\text { (in adduct) }\end{array}$ & Total & \\
\hline $\begin{array}{c}\text { Free BW } 245 C \\
\text { Dextran }(71,200)-B W 245 C\end{array}$ & $\left(2.7 \times 10^{-3}\right)$ & - & 1 \\
\hline $\begin{array}{l}25 \\
2 \cdot 5 \\
0 \cdot 25 \\
0.025\end{array}$ & $\begin{array}{l}8 \cdot 3 \\
0.23 \\
0.13 \\
0.085\end{array}$ & $\begin{array}{r}33 \\
9 \\
51 \\
340\end{array}$ & $\begin{array}{l}3.3 \times 10^{-4} \\
1.2 \times 10^{-2} \\
2.1 \times 10^{-2} \\
3.2 \times 10^{-2}\end{array}$ \\
\hline $\begin{array}{c}P E G(10,000)-B W 245 C \\
0.20 \\
0.01\end{array}$ & $\begin{array}{l}0.055 \\
5.7 \times 10^{-3}\end{array}$ & $\begin{array}{l}27 \cdot 5 \\
57 \cdot 4\end{array}$ & $\begin{array}{l}4.9 \times 10^{-2} \\
0.47\end{array}$ \\
\hline $\begin{array}{c}P E G(4,000)-B W 245 C \\
0.70 \\
0.07 \\
0.035\end{array}$ & $\begin{array}{l}0.14 \\
0.059 \\
0.048\end{array}$ & $\begin{array}{l}19 \cdot 7 \\
84 \cdot 3 \\
137\end{array}$ & $\begin{array}{l}2.0 \times 10^{-2} \\
4.6 \times 10^{-2} \\
5.6 \times 10^{-2}\end{array}$ \\
\hline
\end{tabular}

Table 2. Anti-aggregatory activities of dipyridamole and derivatives.

\begin{tabular}{lcc}
\hline & $\begin{array}{c}\text { Concentration of dipyridamole } \\
\text { (in adduct) required for } 50 \% \\
\text { inhibition }(\mu \mathrm{g} / \mathrm{ml})\end{array}$ & $\begin{array}{c}\text { Relative molar } \\
\text { activity of dipyrida- } \\
\text { mole }\end{array}$ \\
\hline Dipyridamole & 200 & 1 \\
DIMA & 250 & 0.8 \\
NVP-DMA copolymer, & 120 & 1.67 \\
9.5\% w/w dipyridamole & 12 & 16.7 \\
NVP-DIMA copolymer, & & \\
20\%.w/w dipyridamole & & \\
\hline
\end{tabular}

inhibitors. We attribute this to adsorption of the adducts on to the platelet membranes, producing high local concentrations of dipyridamole which interact readily with (external) receptors by virtue of the less severe steric restraint.

A second property of dipyridamole is its ability to potentiate the inhibitory activity of prostaglandins, including BW $245 \mathrm{C}$, especially for aggregation induced by platelet activation factor (PAF-acether). This behaviour is commonly ascribed to the inhibition by dipyridamole of intra-cellular phosphodiesterase, an enzyme which decomposes c-AMP. When dipyridamole is coupled to poly-NVP its potentiating ability is very greatly reduced, or eliminated (Bamford et al 1987a). However, the adduct is just as potent as dipyridamole as a phosphodiesterase inhibitor when assessed in vitro, hence we have concluded that the polymer chains of the adduct prevent penetration of the platelet membrane and so preclude access to intracellular phosphodiesterase (Bamford et al 1987a). A similar type of behaviour has been observed with theophylline (1ㅇ) which, like dipyridamole, is a potentiator of prostaglandin inhibitory activity. We have prepared adducts of theophylline with various polymers, including dextran, PEG and PNVP and found that although (like theophylline) they are phosphodiesterase inhibitors, in no case 
<smiles>Cn1c(=O)c2[nH]cnc2n(C)c1=O</smiles>

do they potentiate BW $245 \mathrm{C}$ activity. This work has been extended to an identification of molecular properties, including size, geometry and the presence of functional groups, which influence membrane penetration; an account will be published separately (Bamford et al 1989).

It is evident from these investigations that the balance of properties of dipyridamole and theophylline is greatly changed when the drugs are coupled to a polymer chain.

The studies with water-soluble polymers described in the preceding pages have relied on simple platelet aggregation. Although they have revealed interesting phenomena-notably the importance of membrane-polymer interactions-they are only a beginning and require extension to other systems and experimental techniques.

\section{References}

Bamford C H 1974 In Reactivity, mechanism and structure in polymer chemistry (eds) A D Jenkins and A Ledwith (New York: Wiley) p. 52

Bamford C H and Middleton I P 1983 Eur. Polym. J. 19 1027; In Plenary and invited lectures Part 2, IUPAC Macro 83, September 5-9, Bucharest, Romania p. 168

Bamford C H, Middleton I P and Al-Lamee K G 1986a Polymer 271981

Bamford C H, Middleton I P and Al-Lamee K G 1986b Biochem. Biophys. Acta 886109

Bamford C H, Middleton I P and Al-Lamee K G 1987a Biochem. Biophys. Acta 92438

Bamford C H, Middleton I P, Al-Lamee K G, Paprotny J and Carr R (in preparation)

Bamford C H, Middleton I P, Al-Lamee K G and Paprotny J 1987b Br. Polym. J. 19269

Bamford C H, Middleton I P, Al-Lamee K G, Paprotny J and Satake Y 1987c Polym. J. $1947 j$

Bamford C H, Middleton I P, Satake Y and Al-Lamee K G 1985 In Advances in polymer synthesis (eds) B M Culbertson and J E McGrath (New York: Plenum) p. 291

Bamford C H, Middleton I P, Satake Y and Al-Lamee K G 1986c In Blood compatible materials and their testing (Dordrecht: Martinus Nijhoff) p. 159

Bots J G F, van der Does L and Bantjes A 1986 Biomaterials 7393

Brockwell M A, Caldwell A G and Whittaker N 1981 J. Chem. Soc., Perkin Trans. 1706

Chesterman C N, Owe-Young R, MacPherson J and Krillis S A 1986 Blood 671744

Clowes A W, Kirkman T R and Reidy M A 1986 Am. J. Pathol. 128220

Ebert C D, Lee E S and Kim S W 1982 J. Biomed. Mater. Res. 16624

Lyman D, Fazzio F I, Voorhees H, Robinson G and Albo D Jr 1978 J. Biomed. Mater. Res. 12337

Ratner B D, Hoffman A S, Hanson S R, Harkes L A and Whiffen J D 1979 J. Polym. Sci., Polym. Symp. 66363

Sa da Costa V, Brier-Russell D, Salzman E W and Merrill E W 1981 J. Coll. Interf. Sci. 80445

Sung C P and Hu C B 1979 J. Biomed. Mater. Res. 13161

Uziel L, Colombo A, Cacciabue E, Cugno M and Agostoni A 1986 In Blood compatible materials and their testing (Dordrecht: Martinus Nijhoff) p. 29

Whittle B J R, Moncada S, Mullane K and Vane J R 1983 Prostaglandins 25205

Wu K K and Hoak J C 1974 Lancet II: 924 